PASQUALE TREMATERRA ${ }^{\mathrm{a}}$ - LORENZO GOGLIA ${ }^{\mathrm{a}}$ - MARCO COLACCI ${ }^{\mathrm{a}}$

\title{
FAUNISTIC NOTES ON LEPIDOPTERA TORTRICIDAE OF GARGANO TERRITORY WITH RECORDING OF ANCYLIS MINIMANA (CARADJA, 1916) NEW TO ITALY
}

\author{
${ }^{a}$ Department of Agriculture, Environmental and Food Sciences, University of Molise, Campobasso, \\ Italy. \\ Corresponding Author: trema@unimol.it
}

Trematerra P., Goglia L., Colacci M. - Faunistic notes on Lepidoptera Tortricidae of Gargano territory with recording of Ancylis minimana (Caradja, 1916) new to Italy.

A list of 67 species of Lepidoptera Tortricidae recorded during several expeditions realized in Gargano territory (Southern Italy) is reported. The two most numerous tortricid subfamilies recorded are the Olethreutinae and Tortricinae, which have 37 species and 30 species, respectively. Species belonging to almost all the tribes cited in the Italian fauna have been found in Gargano territory (Tortricini with 4 species, Cochylini 10, Cnephasiini 6, Archipini 10, Olethreutini 7, Enarmoniini 4, Eucosmini 12, and Grapholitini 14). Ancylis minimana (Caradja, 1916) is reported for the first time in Italy.

KEY WORDS: Lepidoptera Tortricidae, fauna, Gargano, Southern Italy

Gargano territory is a mountainous promontory jutting into the Adriatic Sea from the east coast of Italy, in Puglia (Apulia) region. Called the "spur" of the Italian "boot" (peninsula). From 1991 most of the territory is national park (118,144 ha) (Fig. I). The peninsula is composed entirely of limestone, surrounded by terraces of various geologic periods, and rises to $1,065 \mathrm{~m}$ at Mount Calvo. The Gargano preserves an extraordinary concentration of different habitats: rocky coasts, the big and hot southern valleys rich in rare flower and wildlife species, the central beech woodlands situated at low altitudes (300 $\mathrm{m}$ a.s.1.) and rich in centuries-old specimens, Mediterranean pine forests with Aleppo pines (Pinus halepensis Mill.), with specimens more than 500 years old. Among the most important endemic species there are Cytisus decumbens (Durande) Spach, Satureja fruticosa italica Fiori, Campanula garganica Ten., and Cistus clusii Dunal (BIONDI et al., 2010). The Umbra Forest (mainly beech, Fagus sylvatica L.) is the most famous of the few surviving forest preserves. Updates on plant species of Gargano are recorded in Habitats Directive 92/43/EEC (EUROPEAN COMMISSION, 1992). Later an inventory of new and rare taxa of Gargano is presented and discussed by PERRINO and WAGENSOMMER (2012).

In the past, the promontory played an important role in the connection with the Balkan flora and fauna, as it is demonstrated by the presence of the so-called "transAdriatic" species (ZANGHERI, 1956; FENAROLI, 1966).

Over time, the Lepidoptera Tortricidae from the Gargano territory has received sporadic attention by both local and foreign entomologists. The only specific works refer to some catches taken from 1953 to 1955 by ZANGHERI (1956). From 1991 to 1997 TREMATERRA, SCIARRETTA, and PARENZAN carried out field studies on Southern Italy and cited also some data related to Gargano promontory (TREMATERRA et al., 1997).

In the present paper, we reported a tortricid list as updated and complete as possible for Gargano. For this purpose, in addition to our specimens collected during 2019-2020, we included in this list both the species found in the course of previous entomological researches in that territory and the species cited in literature.

Our specimens were caught both to light at nighttime, mostly using UV-LED light traps and also by butterfly net during day-time. The material was identified morphologically; genitalia were prepared using standard methods. All specimens and slides were deposited in the Trematerra Collection (at the University of Molise, Campobasso, Italy).

In the compilation of this inventory, we followed the systematic arrangement adopted by RAZOWSKI (2002 and 2003) with some modifications suggested by BROWN (2005) and by GILLIGAN et al. (2018). Distribution of the species was largely taken from RAZOWSKI (2002 and 2003), TREMATERRA (2003) and AARVIK (2013), with new data from Trematerra's personal catalogue (unpubl. data). Chorotype of the species was taken from VIGNA TAGLIANTI et al. (1992) and PARENZAN (1994).

\section{LIST OF TAXA}

\section{FAMILY TORTRICIDAE}

\section{SUBFAMILY TORTRICINAE}

\section{Tribe Tortricini}

\section{Aleimma loeflingianum (Linnaeus, 1758)}

MATERIAL EXAMINED. 1 male, Gargano, San Nicandro Garganico (Foggia), 300 m, 8.VI.1991, leg. P. Trematerra. 1 female, Foresta Umbra, 800 m, 6.VIII.1977, Ist. Ent. Bari; 2 females, idem, 12.VI.1982. 

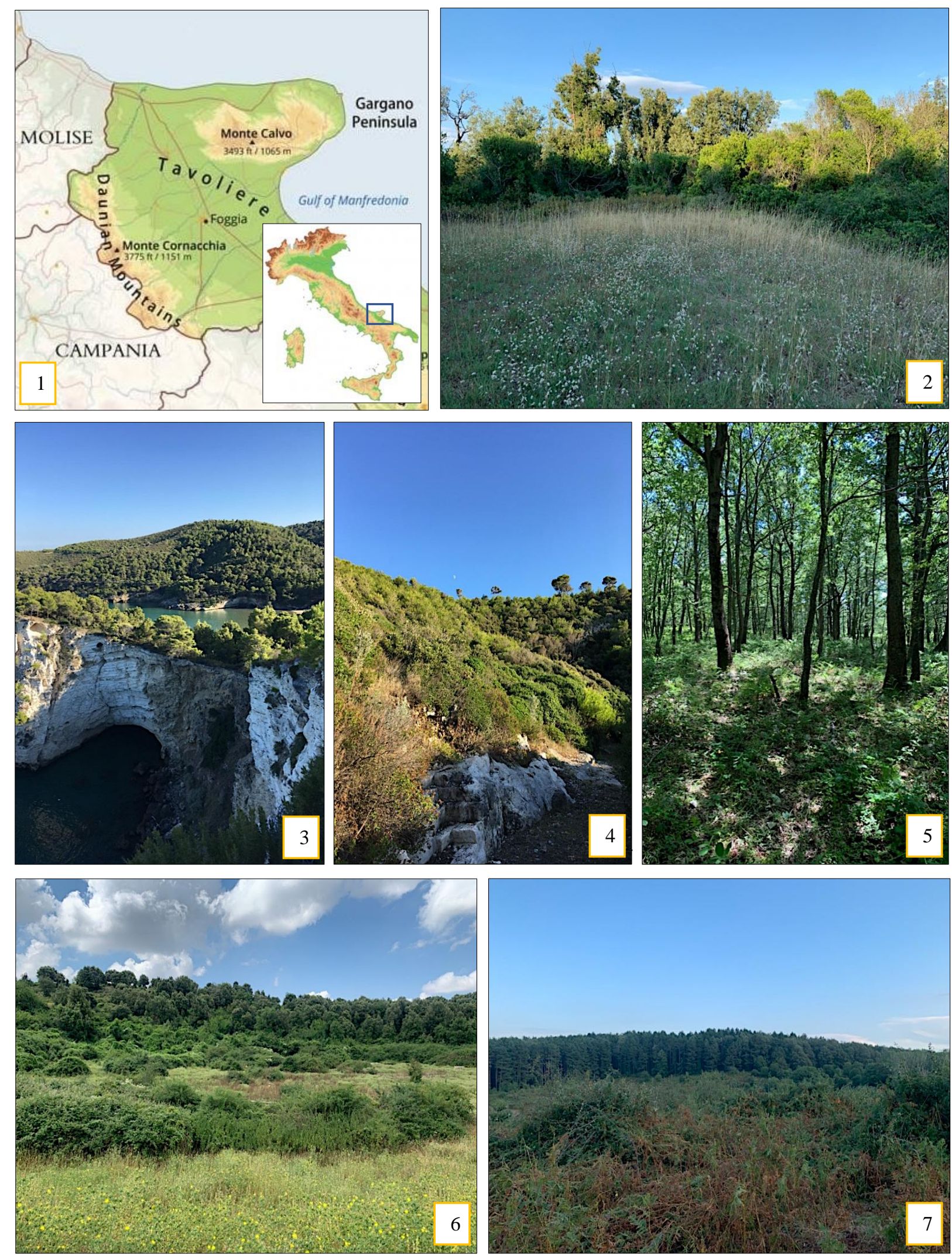

Fig. I - Maps of the Gargano territory (Southern Italy) (1). Some habitas of Gargano territory: Bosco Isola, Lesina, $6 \mathrm{~m}$ a.s.1. (2); Baia San Felice, Vieste, 29 m a.s.l. (3); Monte Pucci, Peschici, 33 m a.s.l. (4); Bosco Spinapulci, San Nicandro Garganico, 650 m a.s.l. (5); Doline Cento Pozzi, Rignano Garganico, 644 m a.s.l. (6); Foresta Umbra, Vico del Gargano, $800 \mathrm{~m}$ a.s.1. (7). 
Distribution. Europe, Ural Mts, Caucasus, Syria, and Asia Minor. (Italy: N, C, S, Si, Sa).

CHOROTYPE. W-Palaearctic.

Acleris forsskaleana (Linnaeus, 1758)

COMMENTS. Reported by ZANGHERI (1956): Bosco Sfilzi, 30.VII.1954, leg. Zangheri.

DisTRIBUTION. Europe. Caucasus, Transcaucasia, Turkmenia, and Iran. Introduced in North America. (Italy: N, C, S, Si, Sa).

CHOROTYPE. Turano-European.

Acleris variegana ([Denis and Schiffermüller], 1775)

MATERIAL EXAMINED. 1 female, Peschici, 29.VI.1980, leg. Baldizzone. 1 female, Vico del Gargano (Foggia), 579 m, 16.VI.2020, leg. Goglia and Palmieri; 1 female, idem, $651 \mathrm{~m}, 18 . \mathrm{VI} .2020$; 1 female, idem, $579 \mathrm{~m}$, 30.VI.2020.

Distribution. From W-Europe and NW-Africa to Iran, Turkmenia, Afghanistan, S-W Siberia, China, and Japan. Present also in North America. (Italy: N, C, S, Si, $\mathrm{Sa})$.

CHOROTYPE. Holarctic.

Acleris hastiana (Linnaeus, 1758)

MATERIAL EXAMINED. 1 male, Lesina (Foggia), 2 m, 18.VI.2020, leg. Goglia and Palmieri.

DisTRIBUTION. A Holarctic species known from WEurope to Japan, Morocco, Siberia, and North America south as far California. (Italy: N, C, S, Sa).

CHOROTYPE. Holarctic.

\section{Tribe Cochylini}

Hysterophora maculosana (Haworth, [1811])

MATERIAL EXAMINED. 1 male and 1 female, San Giovanni Rotondo (Foggia), 600 m, 16.IV.1997, leg. Mancini, Sciarretta, Trematerra.

DisTRIBUTION. Europe, Israel, Asia Minor, and Transcaucasia. (Italy: N, C, S, Si, Sa).

CHOROTYPE. Turano-European.

Phalonidia contractana (Zeller, 1874)

MATERIAL EXAMINED. 1 female, Lesina (Foggia), 2 m, 2.VII.2020, leg. Goglia and Palmieri.

DISTRIBUTION. Widely distributed from Iberian Peninsula to Ural Mts; found also in N-Africa, Asia Minor, Israel, Transcaucasia, Iran, Afghanistan, Kashmir, and Central Asia. (Italy: N, C, S, Si, Sa).

CHOROTYPE. Centralasiatic-Europeo-Mediterranean.

Gynnidomorpha rubricana (Peyerimhoff, 1877)

MATERIAL EXAMINED. 1 female, Vico del Gargano (Foggia), 559 m, 1.VII.2020, leg. Goglia and Palmieri.

DisTRIBUTION. Known from SE-Europe, China,

Russian Far East, Korea, and Japan. (Italy: N, C, S).

CHOROTYPE. Asiatic-European.

Aethes tesserana ([Denis and Schiffermüller], 1775) MATERIAL EXAMINED. 1 female, Foresta Umbra
(Foggia), 700 m, 16.IV.1997, leg. Mancini, Trematerra, Sciarretta.

COMMENTS. Reported by ZANGHERI (1956), Foresta Umbra, 14.VII.1954.

DISTRIBUTION. Europe, Asia Minor, Transcaucasia, Iran, and S-Siberia. (Italy: N, C, S, Si, Sa).

ChOROTYPE. Centralasiatic-European.

Aethes francillana (Fabricius, 1794)

MATERIAL EXAMINED. 1 female, Rignano Garganico (Foggia), 629 m, 16.VII.2020, leg. Goglia and Palmieri.

COMMENTS. Reported by ZANGHERI (1956), Foresta Umbra, 14-28.VII.1954.

DistriBUTION. Europe, Canary Islands and NWAfrica to Transcaucasia, Iran, Afghanistan, Kazakhstan, Tadzhikistan, and W-Siberia. (Italy: N, C, S, Si, Sa).

CHOROTYPE. W-Palaearctic.

Diceratura roseofasciana (Mann, 1855)

MATERIAL EXAMINED. 19 males and 2 females, Lesina (Foggia), 5 m, 15.VII.2020, leg. Goglia and Palmieri. 1 male, Vico del Gargano (Foggia), $558 \mathrm{~m}$, 29.VII.2020, leg. Goglia and Palmieri.

Distribution. Europe, Asia Minor, Near East, Iran, Transcaucasia, and Kazakhstan. (Italy: C, S, Si, Sa). CHOROTYPE. Turano-European.

Diceratura ostrinana (Guenée, 1845)

MATERIAL EXAMINED. 1 male, Gargano, Carpino (Foggia), 300-500 m, 16.IV.1997, leg. Mancini, Sciarretta, Trematerra.

COMMENTS. Listed by ZANGHERI (1956), 14 15.VII.1954.

DisTRIBUTION. West Palaearctic species known from Algeria and Iberian Peninsula to Hungary and Albania, Greece and Crimea. Other records are from Lebanon, Asia Minor, Transcaucasia, and Siberia. (Italy: N, $\mathrm{C}, \mathrm{S}, \mathrm{Sa})$.

CHOROTYPE. Centralasiatic-European.

Cochylis hybridella (Hübner, [1813])

MATERIAL EXAMINED. 3 males, Foresta Umbra, 800 m, 6.VIII.1977, Ist. Ent. Bari.

COMMENTS. Reported by ZANGHERI (1956): Foresta Umbra, 16.VII.1954, leg. Zangheri.

DISTRIBUTION. Europe, Asia Minor, Transcaucasia, Ural Mts, Kazakhstan, W-S Siberia, China, Russian Far

East, Korea, and Japan. (Italy: N, C, S, Si, Sa).

CHOROTYPE. Asiatic-European.

Cochylis molliculana Zeller, 1847

MATERIAL EXAMINED. 1 male, Capoiale (Foggia), 2 m, 16.VI.2020, leg. Goglia and Palmieri. 1 male, Lesina (Foggia), 2 m, 18.VI.2020, leg. Goglia and Palmieri; 1 male, idem, 2 m, 1.VII.2020; 1 male, idem, 2.VII.2020. 1 male, San Nicandro Garganico (Foggia), 649 m, 30.VI.2020, leg. Goglia and Palmieri.

DisTRIBUTION. Great Britain and from Spain to Greece. (Italy: N, C, S, Si).

CHOROTYPE. Europeo-Mediterranean 
Cochylis posterana Zeller, 1847

MATERIAL EXAMINED. 2 females, Foresta Umbra, 800 m, 6.VIII.1977, Ist. Ent. Bari. 3 males, Vico del Gargano (Foggia), 559 m, 1.VII.2020, leg. Goglia and Palmieri; 4 males, idem, 538 m, 28.VII.2020; 1 male, idem, $558 \mathrm{~m}, 29$.VII. 2020.

COMMENTS. Reported by ZANGHERI (1956): Foresta Umbra, 20-28.VII.1954, leg. Zangheri.

DISTRIBUTION. W-Palaearctic species occurring east far as Transcaspia, Central Asia and W-Siberia. (Italy: N, C, S, Si, Sa).

CHROTYPE. Palaearctic.

\section{Tribe Cnephasiini}

Propiromorpha rhodophana (Herrich-Schäffer, 1851)

MATERIAL EXAMINED. 1 male, Lesina (Foggia), 4 m, 17.VI.2020, leg. Goglia and Palmieri. 1 male, Vico del Gargano (Foggia), 559 m, 1.VII.2020, leg. Goglia and Palmieri.

DistRIBUTION. S-Europe, NW-Africa, Asia Minor, Mongolia, China, Amur Territory, and Primorsk. (Italy: $\mathrm{N}, \mathrm{C}, \mathrm{S})$

CHOROTYPE. Palaearctic.

Cnephasia incertana (Treitschke, 1835)

MATERIAL EXAMINED. 1 male, San Nicandro Garganico (Foggia), 300 m, 8.VI.1991, leg. P. Trematerra. 1 male, Torre Mileto (Foggia), 30 m, 9.IV.1992, leg. P. Trematerra. 3 males, Monte Sant'Angelo (Foggia), 500 m, 16.IV.1997, leg. Mancini, Sciarretta, Trematerra.

DisTRIBUTION. Western Palaearctic from Iberian Peninsula to Transcaucasia. (Italy: N, C, S, Si, Sa).

CHOROTYPE. W-Palaearctic.

Cnephasia asseclana ([Denis and Schiffermüller], 1775)

COMMENTS. Reported by ZANGHERI (1956): Monte S. Angelo, 20.V.1954, leg. Zangheri; Foresta Umbra, 14.VII.1954, leg. Zangheri.

DisTRIBUTION. Europe, Canary Islands and New Foundland, Morocco, Caucasus, Transcaucasia, W-S Siberia, and Japan. Introduced to North America. (Italy: N, C, S).

CHOROTYPE. Holarctic.

Cnephasia pasiuana (Hübner, [1799])

MATERIAL EXAMINED. 1 male, Gargano, Monte Delio (Foggia), 50 m, 8.VI.1991, leg. P. Trematerra.

DisTRIBUtion. Europe, Morocco, Egypt, Asia Minor, Syria, Libya, and Transcaucasia. (Italy: N, C, S, Si).

CHOROTYPE. W-Palaearctic.

Cnephasia abrasana (Duponchel, 1843)

MATERIAL EXAMINED. 1 male and 1 female, Torre Mileto (Foggia), 30 m, 9.VI.1992, leg. P. Trematerra. 2 males and 1 female, Monte Sant'Angelo (Foggia), $500 \mathrm{~m}$, 16.VI.1997, leg. Mancini, Sciarretta, Trematerra. 1 male, Gargano (Foggia), 300-500 m, 16.VI.1997, leg. Mancini, Sciarretta, Trematerra.

DistRIBUTION. Europe and Asia Minor. (Italy: N, $\mathrm{C}, \mathrm{S}, \mathrm{Si}, \mathrm{Sa})$.
CHOROTYPE. European.

Cnephasia ecullyana Réal, 1951

MATERIAL EXAMINED. 2 males, San Nicandro Garganico (Foggia), 300 m, 8.VI.1991, leg. Trematerra. 1 male, Torre Mileto (Foggia), 30 m, 9.V.1992, leg. Trematerra. 1 male, Vico del Gargano (Foggia), $579 \mathrm{~m}$, 16.VI.2020, leg. Goglia and Palmieri; 2 females, idem, $559 \mathrm{~m}, 1$. VII.2020.

DisTRIBUTION. Known from Central Southern Europe. (Italy: N, C, S, Si, Sa).

CHOROTYPE. S-European.

\section{Tribe Archipini}

Batodes angustiorana (Haworth, [1811])

COMMENTS. Reported by ZANGHERI (1956): Foresta Umbra, 28.VII.1954.

DisTRIBUTION. Europe, NW-Africa, and Asia Minor. Introduced to North America. (Italy: N, C, S, Sa).

CHOROTYPE. Holarctic.

Epagoge grotiana (Fabricius, 1781)

MATERIAL EXAMINED. 1 female, San Nicandro Garganico (Foggia), 649 m, 30.VI.2020, leg. Goglia and Palmieri.

COMMENTS. Reported by ZANGHERI (1956): Foresta Umbra, 14.VIII.1954, leg. Zangheri.

DISTRIBUTION. Distributed in W-Palaearctic from WN-Europe to Ukraine and S-Ural Mts, Trans-Ural, Caucasus, and Asia Minor. (Italy: N, C, S, Si).

Chorotype. W-Palaearctic.

Archips crataeganus (Hübner, [1799])

MATERIAL EXAMINED. 1 female, San Nicandro Garganico (Foggia), 649 m, 30.VI.2020, leg. Goglia and Palmieri.

DisTRIBUTION. W-Palaearctic, Kazakhstan, Siberia, China, Korea, and Japan. (Italy: N, C, S).

ChOROTYPE. Palaearctic.

Archips xylosteanus (Linnaeus, 1758)

MATERIAL EXAMINED. 1 male, Monte Delio (Foggia), 50 m, 8.VI.1991, leg. P. Trematerra.

COMMENTS. Reported by ZANGHERI (1956): Bosco Ginestra, IX.1954, leg. Zangheri.

Distribution. Palaearctic Region from W-Europe to Asia Minor, Caucasus, Iran, Central Asia, China, Russian Far East, and Japan. (Italy: N, C, S, Si, Sa).

CHOROTYPE. Asiatic-European.

Argyrotoenia ljungiana (Thunberg, 1797)

MATERIAL EXAMINED. 1 male, Vico del Gargano (Foggia), 579 m, 16.VI.2020, leg. Goglia and Palmieri; 3 females, idem, $559 \mathrm{~m}, 1$.VII.2020.

DISTRIBUTION. Palaearctic species distributed from Iberian Peninsula and NW-Africa as far as China, Russian Far East, and North America. (Italy: N, C, S).

CHOROTYPE. Holarctic.

Cacoecimorpha pronubana (Hübner, [1799])

MATERIAL EXAMINED. 1 male, Vico del Gargano 
(Foggia), 559 m, 1.VII.2020, leg. Goglia and Palmieri. Distribution. Western Palaearctic from Europe, Morocco, Algeria and Tunisia to Lebanon, Asia Minor, Near East, Crimea, and Japan. Introduced to South Africa and North America. (Italy: N, C, S, Si, Sa).

CHOROTYPE. Cosmopolitan.

Aphelia ferrugana (Hübner, 1793)

COMMENTS. Reported by ZANGHERI (1956):

Foresta Umbra, 21.V.1954, leg. Zangheri.

Distribution. Europe, Caucasus, Asia Minor, Syria, Iraq, and Iran. (Italy: N, C, S, Si, Sa).

CHOROTYPE. Turano-European.

Clepsis unicolorana (Duponchel, 1835)

COMMENTS. Reported by ZANGHERI (1956):

Foresta Umbra, 23.V.1954, leg. Zangheri.

DistRIBUTION. Known from Europe (Portugal, Spain, France, Belgium, Italy, Switzerland, Malta), to Morocco and Mongolia. (Italy: N, C, S).

CHOROTYPE. Centralasiatic-Europeo-Mediterranean.

Clepsis dumicolana (Zeller, 1847)

COMMENTS. Reported by TREMATERRA et al. (1997): Foresta Umbra, 800 m, 6.VIII.1977.

Distribution. S-Europe, Asia Minor, Syria, and Lebanon. (Italy: N, C, S, Si, Sa).

CHOROTYPE. S-European-Anatolic.

Clepsis consimilana (Hübner, [1817])

MATERIAL EXAMINED. 7 males e 1 female, Vico del Gargano (Foggia), 579 m, 16.VI.2020, leg. Goglia and Palmieri; 1 male, idem, $693 \mathrm{~m}, 14$. VII.2020; 1 male, idem, 579 m, 19.VIII.2020, leg. Palmieri. 2 males e 4 females, Lesina (Foggia), 4 m, 17.VI.2020, leg. Goglia and Palmieri; 1 male, idem, 2 m, 18.VI.2020. 1 male, San Nicandro Garganico (Foggia), 651 m, 18.VI.2020, leg. Goglia and Palmieri. 3 males e 5 females, Vieste (Foggia), 33 m, 15.VII.2020, leg. Goglia and Palmieri. 1 male, Rignano Garganico (Foggia), 629 m, 16.VII.2020, leg. Goglia and Palmieri. 1 female, San Giovanni Rotondo (Foggia), 595 m, 25.VIII.2020, leg. Goglia and Palmieri. 17 males e 3 females, Peschici (Foggia), 20 m, 26.VIII.2020, leg. Goglia and Palmieri.

COMMENTS. Reported by ZANGHERI (1956): Foresta Umbra, 14-15.VII.1954, leg. Zangheri.

DisTRIBUTION. W-Europe to E-European Russia; W-Africa to Lebanon, Asia Minor, Nearctics and Ethiopian region (introduced). (Italy: N, C, S, Si, Sa).

CHOROTYPE. Cosmopolitan.

\section{SUBFAMILY OLETHREUTINAE}

Tribe Olethreutini

Endothenia gentianaeana (Hübner, [1799])

MATERIAL EXAMINED. 1 male, Vico del Gargano

(Foggia), 536 m, 28.VII.2020, leg. Goglia and Palmieri.
COMMENTS. Reported by ZANGHERI (1956): Foresta Umbra, 15.VII.1954, leg. Zangheri.

Distribution. Europe to Ural Mts, Asia Minor, Arabian Peninsula, Transcaucasia, C-Asia, Mongolia, SSiberia, China, Korea, and Japan. (Italy: N, C, S, Si).

CHOROTYPE. Asiatic-European.

Endothenia marginana (Haworth, [1811])

MATERIAL EXAMINED. 1 female, Foresta Umbra, 800 m, 6.VIII.1977, Ist. Ent. Bari. 1 male, Lesina (Foggia), 4 m, 17.VI.2020, leg. Goglia and Palmieri; 1 male, idem, 2 m, 2.VII.2020. 2 males, San Nicandro Garganico (Foggia), 649 m, 30.VI.2020, leg. Goglia and Palmieri. 3 males e 1 female, Vico del Gargano (Foggia), $559 \mathrm{~m}$, 1.VII.2020, leg. Goglia and Palmieri; 1 male, idem, 536 m, 6.VII.2020; 2 males, idem, 28.VII.2020.

DistRIBUTION. Europe, NW-Africa, Transcaucasia, Asia: from Ural Mts to Mongolia, China and the Russian Far East. (Italy: N, C, S, Si, Sa).

CHOROTYPE. Palaearctic.

Lobesia botrana ([Denis and Schiffermüller], 1775) MATERIAL EXAMINED. 5 males and 1 female, Lesina (Foggia), 4 m, 17.VI.2020, leg. Goglia and Palmieri; 2 males and 3 females, idem, 2 m, 18.VI.2020; 9 males, idem, 2 m, 2.VII.2020. 1 male, San Nicandro Garganico (Foggia), 649 m, 30.VI.2020, leg. Goglia and Palmieri. 2 males, Vico del Gargano (Foggia), 559 m, 1.VII.2020, leg. Goglia and Palmieri; 4 males, idem, $538 \mathrm{~m}$, 28.VII.2020; 16 males and 1 female, idem, $558 \mathrm{~m}$, 29.VII.2020; 3 males, idem, 536 m, 6.VIII.2020.

DisTRIBUTION. From W-Europe to Ural Mts, N-Africa, Asia Minor, Near East, Iraq, Iran, Transcaucasia, Turkmenistan, Kazakhstan, and Japan. N-S America (introduced). (Italy: N, C, S, Si, Sa).

CHOROTYPE. Cosmopolitan.

Hedya nubiferana (Haworth, [1811])

MATERIAL EXAMINED. 1 male, San Nicandro Garganico (Foggia), 649 m, 30.VI.2020, leg. Goglia and Palmieri.

DisTRIBUTION. W-Europe to Ural Mts, Caucasus, Asia Minor, Iraq, Turkmenistan, Iran, Kazakhstan, W-Siberia. North America (Canada). (Italy: N, C, S, Si, Sa).

CHOROTYPE. Holarctic.

Hedya pruniana (Hübner, [1799])

MATERIAL EXAMINED. 2 males and 1 female, Monte Sant'Angelo, Vallone Pulsano (Foggia), $400 \mathrm{~m}$, 16.IV.1997, leg. Mancini, Sciarretta, Trematerra.

DisTRIBUTION. From W-Europe to Ural Mts, Asia Minor, Caucasus, Kazakhstan, N-Iran, and Russian Far East. (Italy: N, C, S, Si, Sa).

CHOROTYPE. Asiatic-European.

Olethreutes arcuellus (Clerck, 1759)

MATERIAL EXAMINED. 1 female, Bosco Quarto (Foggia), 600 m, 16.IV.1997, leg. Mancini, Sciarretta, Trematerra. 1 female, Foresta Umbra (Foggia), 700 m, 16.IV.1997, leg. Mancini, Sciarretta, Trematerra. 

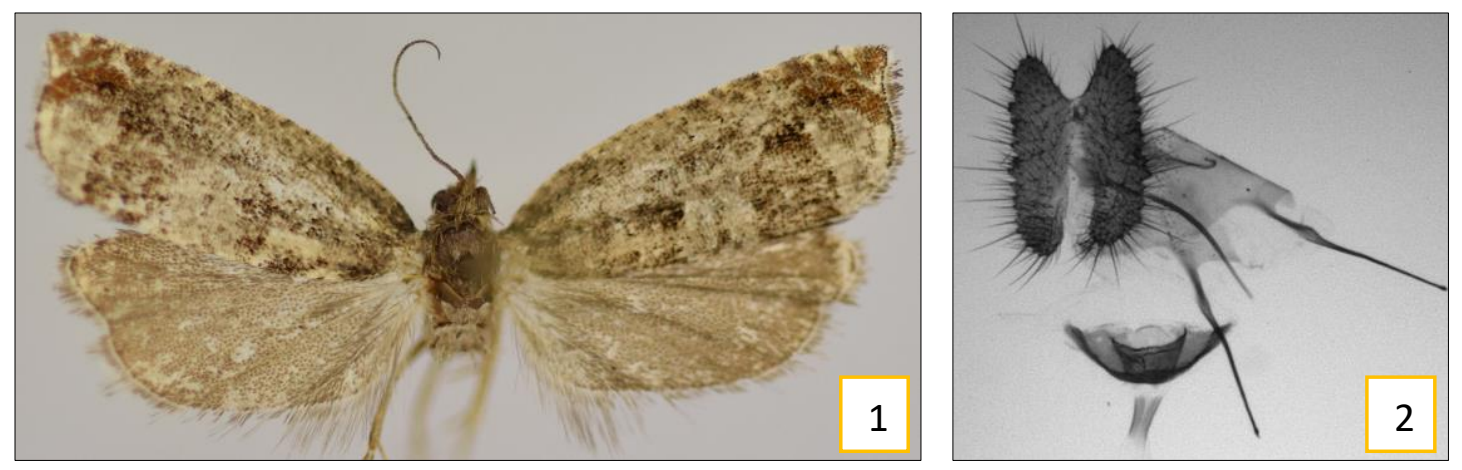

Fig. II - Ancylis minimana (Caradja): adult (1); female genitalia (papille anales, antrum, sterigma, and cingulum) (2).

COMMENTS. Reported by ZANGHERI (1956):

Foresta Umbra, 21-23.V.1954, leg. Zangheri.

DISTRIBUTION. From Europe to Caucasus; Asia MiMinor, Iran, Kazakhstan, W-Siberia, and NW-China. (Italy: N, C, S, Si).

CHOROTYPE. Centralasiatic-European.

Syricoris lacunana ([Denis and Schiffermüller], 1775) MATERIAL EXAMINED. 2 males, Vico del Gargano (Foggia), 693 m, 14.VII.2020, leg. Goglia and Palmieri. DisTRIBUTION. W-Europe to Ural Mts and Caucasus, Transcaucasia; Asia Minor, Mongolia, WS-Siberia, China, and Japan. (Italy: N, C, S, Sa).

CHOROTYPE. Asiatic-European.

Tribe Enarmoniini

Ancylis comptana (Frölich, 1828)

Material EXAMINed. 1 female, Peschici, 29.VI.1980, leg. Baldizzone.

DisTRIBUTION. From Europe to Ural Mts, Asia Minor, Caucasus, Kazakhstan, Uzbekistan, Siberia Far East, China, Mongolia, Korea, and Japan. Also North America. (Italy: N, C, S, Sa).

CHOROTYPE. Holarctic

Ancylis minimana (Caradja, 1916)

MATERIAL EXAMINED. 1 female, Vico del Gargano (Foggia), 579 m, 16.VI.2020, leg. Goglia and Palmieri; 1 female, idem, $559 \mathrm{~m}$, 1.VII.2020.

DESCRIPTION. Adult wing span 10-12 mm (Fig. II, 1). Ground colour of forewing whitish grey distinctly suffused grey-brown especially in terminal part of wing; costal strigulae whitish, dividings brown; postbasal interfascia and area of speculum slightly paler than markings. Markings brown or greyish brown. Distal edge of dorsobasal blotch weakly convex; median fascia slender in costal half. Cilia whitish with brownish or grey parts. Hindwing grey with brownish admixture; cilia much paler.

In male genitalia uncus rudimentary in form of terminal lobe of tegumen; socius broad, rounded; sacculus with sharp ventro-posterior prominence; neck of valva slender, rather short; cucullus ovate, with atrophying ventral lobe; aedeagus slender.

In female genitalia the postostial part of the sterigma is rather large; cingulum median; the signa pair are large and rather equal (Fig. II, 2).

DISTRIBUTION. From Eastern and SE parts of E-Europe to Ciscaucasia and Ural Mts, Caucasus. (Italy: Puglia).

CHOROTYPE. Turano-European.

BIOLOGY. Moth collected in June-July. Larva found on buckthorn Hippophae spp.

REMARKS. New to the Italian fauna. Ancylis minimana is reported by RAZOWSKI (2003) among Ancylis myrtillana (Treitschke, 1830) and Ancylis apicella ([Denis and Schiffermüller], 1775).

Ancylis badiana ([Denis and Schiffermüller], 1775)

MATERIAL EXAMINED. 1 female, Foresta Umbra, 800 m 6.VIII.1977, Ist. Ent. Bari.

COMMENTS. Reported by ZANGHERI (1956): Foresta Umbra, 21.V.1954, leg. Zangheri.

Distribution. From W-Europe to Asia Minor, Caucasus, Transcaucasia, Ural Mts, Mongolia and Russian Far East, China, Korea, and Japan. (Italy: N, C, S, $\mathrm{Si}, \mathrm{Sa}$ ).

CHOROTYPE. Asiatic-European.

Enarmonia formosana (Scopoli, 1763)

COMMENTS. Reported by ZANGHERI (1956): Bosco Sfilzi, 18.VII.1954, leg. Zangheri.

Distribution. Europe, N-Africa, Ural Mts, Asia Minor, Transural, Caucasus, Kazakhstan, Russian Far East, Japan. Canada (introduced?). (Italy: N, C, S, Si, Sa). CHOROTYPE. Holarctic.

\section{Tribe Eucosmini}

Thiodia trochilana (Frölich, 1828)

MATERIAL EXAMINED. 1 male and 1 female, Lesina (Foggia), Bosco Isola, 3.VII.1980, leg. Baldizzone. 1 male, Gargano, Torre Mileto (Foggia), 30 m, 9.IV.1992, leg. P. Trematerra. 
DISTRIBUTION. CS-Europe, East Balticum, southern part of E-Europe, N-Africa, Asia Minor, Transcaucasia, Turkmenia, and Iran. (Italy: N, C, S, Si).

Chorotype. Turano-Europeo-Mediterranean.

Rhopobota naevana (Hübner, [1817])

COMMENTS. Reported by ZANGHERI (1956): Foresta Umbra, 20-31.VII.1954, leg. Zangheri.

DisTRIBUTION. From Europe to Ural Mts, Caucasus, Transcaucasia, India, Sri-Lanka, Siberia, Far East, Mongolia, China, Korea, and Japan. In the Nearctic Region occurs ssp. illicifoliana Kearfott, 1907. (Italy: N, C, S, Si, $\mathrm{Sa})$.

ChOROTYPE. Subcosmopolitan.

Spilonota ocellana ([Denis and Schiffermüller], 1775) MATERIAL EXAMINED. 2 males, Vico del Gargano (Foggia), 536 m, 28.VII.2020, leg. Goglia and Palmieri.

COMMENTS. Reported by ZANGHERI (1956): Foresta Umbra, 20.VII. 1954, leg. Zangheri; Bosco Sfilzi, 18.VII.1954, leg. Zangheri.

DisTRIBUTION. Europe, N-Africa, Caucasus, Asia Minor, Transcaucasia, Iran, Kazakhstan, Turkmenia, Uzbekistan, Tadzhikistan, Russian Far East, Siberia, China, Korea, and Japan. Also Nearctic Region. (Italy: N, C, S, $\mathrm{Si}, \mathrm{Sa})$.

CHOROTYPE. Holarctic.

Crocidosema plebejana Zeller, 1847

MATERIAL EXAMINED. 1 female, Lesina (Foggia), 2 m, 2.VII.2020, leg. Goglia and Palmieri. 1 female, Vico del Gargano (Foggia), 558 m, 29.VII.2020, leg. Goglia and Palmieri.

DistRIBUTION. Entire Europe, N-Africa, Asia Minor, Arabia, Iran, Transcaucasia, Turkmenia, India, Taiwan, Australia, North America, and South America. (Italy: N, C, S, Si, Sa).

CHOROTYPE. Cosmopolitan.

Pelochrista fusculana (Zeller, 1847)

MATERIAL EXAMINED. 4 males and 2 females, Torre Mileto (Foggia), 30 m, 9.IV.1992, leg. P. Trematerra.

DiSTRIBUTION. S-Europe from Spain to former $\mathrm{Yu}$ goslavia, Bulgaria and Romania. NW-Africa, (Italy: N, $\mathrm{C}, \mathrm{S}, \mathrm{Si}, \mathrm{Sa})$.

CHOROTYPE. S-European.

Pelochrista agrestana (Treitschke, 1830)

MATERIAL EXAMINED. 1 male, Foresta Umbra, 600 m, 4.VII.1980, leg. Baldizzone. 1 male, Vico del Gargano (Foggia), 536 m, 28.VII.2020, leg. Goglia and Palmieri; 1 male and 1 female, idem, 6.VIII.2020.

DISTRIBUTION. Known chiefly from Lithuania, SWEurope (France, Italy, Austria and former Yugoslavia), and Asia Minor. (Italy: C, S, Si).

CHOROTYPE. S-European.

Eucosma lugubrana (Treitschke, 1830)

COMMENTS. Reported by ZANGHERI (1956) as present in Gargano promontory.

DISTRIBUTION. Europe, Kazakhstan, Kyrgyzstan,
Russian (W-Tuva). (Italy: N, S, Si).

CHOROTYPE. Centralasiatic-European.

Eucosma cana (Hawort, [1811])

MATERIAL EXAMINED. 1 male, Vico del Gargano (Foggia), 693 m, 14.VII.2020, leg. Goglia and Palmieri.

DistRIBUTION. Europe, Asia Minor, Caucasus, Transcaucasia, Kazakhstan, C-Asia, S-Siberia, Russian Far East, China, and Japan. (Italy: N, C, S, Si, Sa).

CHOROTYPE. Asiatic-European.

Eucosma lacteana (Treitschke, 1835)

MATERIAL EXAMINED. S. Nicola, Varano, 23.V.1992, leg. G.R. Langohr.

DistrIBUTION. From Europe to Russian Far East, SW-Siberia, and Mongolia. (Italy: N, C, S).

CHOROTYPE. Sibero-European.

Notocelia cynosbatella (Linnaeus, 1758)

MATERIAL EXAMINED. 1 male, Carpino (Foggia), 300-500 m, 16.V.1997, leg. Mancini, Sciarretta, Trematerra. 1 female, Monte Sant'Angelo (Foggia), Vallone Pulsano, 400 m, 16.V.1997, leg. Mancini, Sciarretta, Trematerra. 1 female, Rignano Garganico (Foggia), 629 m, 16.VII.2020, leg. Goglia and Palmieri.

DisTRIBUTION. Europe, Ural Mts, Near East, Caucasus, Transcaucasia, Asia Minor, Iran, Kazakhstan, Central Asia, Mongolia, Siberia, and Russian Far East. (Italy: N, C, S, Si).

CHOROTYPE. Asiatic-European.

Notocelia uddmanniana (Linnaeus, 1758)

MATERIAL EXAMINED. 2 males, San Nicandro Garganico (Foggia), $651 \mathrm{~m}, 18$. VI.2020, leg. Goglia and Palmieri; 2 males and 1 female, idem, 649 m, 30.VI.2020. 2 males, Vico del Gargano (Foggia), 538 m, 28.VII.2020, leg. Goglia and Palmieri.

COMMENTS. Reported by ZANGHERI (1956): Foresta Umbra, 14-29.VII.1954, leg. Zangheri.

DisTRIBUTION. Europe, N-Africa, Asia Minor, Near east, Caucasus, Transcaucasia, Transural, Iran, Kazakhstan, Tadzhikistan, and S-Siberia. (Italy: N, C, S, Si, Sa). CHOROTYPE. Palaearctic.

Notocelia aquana (Hübner, [1799])

COMMENTS. Reported by ZANGHERI (1956): Foresta Umbra, 14-21.VII.1954, leg. Zangheri.

DistribUtion. W-Europe, Morocco, Ural Mts, Caucasus, Transcaucasia, Asia Minor, Iran, Kazakhstan, C-Asia, W-Siberia, and China. (Italy: N, C, S, Si). CHOROTYPE. Palaearctic.

Tribe Grapholitini

Cydia semicinctana (Kennel, 1901)

MATERIAL EXAMINED. 2 males, Vico del Gargano (Foggia), 559 m, 1.VII.2020, leg. Goglia and Palmieri; 1 male, idem, 536 m, 28.VII.2020.

DisTRIBUTION. Locally in Europe, Asia Minor; NIran, Uzbekistan, and Turkmenia. (Italy: N, C, S).

CHOROTYPE. Turano-European. 
Cydia pomonella (Linnaeus, 1758)

MATERIAL EXAMINED. 2 males, Vico del Gargano (Foggia), 559 m, 1.VII.2020, leg. Goglia and Palmieri; 3 males, idem, 558 m, 29.VII.2020. 1 male, Lesina (Foggia), 2 m, 2.VII.2020, leg. Goglia and Palmieri.

COMMENTS. Reported by ZANGHERI (1956): Foresta Umbra, 28.VII.1954, leg. Zangheri.

DisTRIBUTION. Covers the area of distribution of wild apple-tree, Europe, Asia, NS-America, Australia, and New Zealand. (Italy: N, C, S, Si, Sa).

CHOROTYPE. Cosmopolitan.

\section{Cydia triangulella (Goeze, 1783)}

MATERIAl EXAMINED. 1 female, Rignano Garganico (Foggia), Dolina Cento Pozzi, 635 m, 20.VI.2020, from Quercus ilex acorns coll. 31.XII.2019, leg. Palmieri.

DisTRIBUTION. Paleartcic region till Japan, its area covers that of oaks. (Italy: N, C, S, Si, Sa).

CHOROTYPE. Palaearctic.

Cydia fagiglandana (Zeller, 1841)

MATERIAL EXAMINED. 1 female, Foresta Umbra, 800 m, 5.VIII.1977, leg. Baldizzone. 1 male, Italia, Puglia, San Nicandro Garganico (Foggia), $540 \mathrm{~m}$, 12.III.2020, from Quercus ilex acorns coll. 24.XII.2019, leg. Palmieri; 1 male, idem, 31.III.2020; 1 male, idem, 27.IV.2020, coll. 3.XI.2019; 1 male, idem, 7.V.2020, coll. 3.XI.2019; 1 female, idem, 9.V.2020, coll. 3.XI.2019. 1 female, Italia, Puglia, Lesina (Foggia), $3 \mathrm{~m}$, 14.III.2020, from Quercus ilex acorns, coll. 1.I.2020, leg. Palmieri. 1 female, Italia, Puglia, San Giovanni Rotondo (Foggia), Pantano di Sant'Egidio, 768 m, 31.V.2020, from Quercus ilex acorns coll. 16.XII.2019, leg. Goglia and Palmieri. 1 male, Lesina (Foggia), 4 m, 17.VI.2020, leg. Goglia and Palmieri; 2 males, idem, $5 \mathrm{~m}$, 15.VII.2020. 1 male and 1 female, Italia, Puglia, Vico del Gargano (Foggia), 693 m, 14.VII.2020, leg. Goglia and Palmieri.

COMMENTS. Reported by ZANGHERI (1956): Foresta Umbra, 19-21.VII.1954, leg. Zangheri; Bosco Sfilzi, 18.VII.1954, leg. Zangheri.

DISTRIBUTION. Europe, Madeira, NW-Africa, Asia Minor, Syria, Iran, and Caucasus. (Italy: N, C, S, Si, Sa). CHOROTYPE. W-Palaearctic.

Cydia inquinatana (Hübner, [1799])

MATERIAL EXAMINED. 1 female, Foresta Umbra, 600 m, 4.VII.1980, leg. Baldizzone.

DiSTRIBUTION. Known from Sweden, Finland, Norway, Russia (St Petersburg), Central and Southern Europe, as far as to lower Volga River territory, Crimea and Caucasus. (Italy: N, C, S, Si).

CHOROTYPE. Europeo-Anatolic.

Lathronympha strigana (Fabricius, 1775)

COMMENTS. Reported by ZANGHERI (1956): Foresta Umbra, 14-15.VII.1956, leg. Zangheri.

Distribution. Europe, Caucasus, Asia Minor and Siberia. (Italy: N, C, S, Si, Sa).

CHOROTYPE. Sibero-European.
Grapholita fissana (Frölich, 1828)

MATERIAL EXAMINED. 1 male, Foresta Umbra, 700 m, 16.V.1999, leg. Mancini, Sciarretta, Trematerra.

DisTRIBUTION. Known from Europe except for British islands and N-Scandinavia, east to Ural Mts, Ciscaucasia and Transcaucasia; also Asia Minor. (Italy: N, $\mathrm{C}, \mathrm{S}, \mathrm{Si}, \mathrm{Sa})$.

CHOROTYPE. Europeo-Anatolic.

Grapholita orobana Treitschke, 1830

MATERIAL EXAMINED. 1 male, Carpino (Foggia), 300-500 m, 16.V.1997, leg. Mancini, Sciarretta, Trematerra.

DisTRIBUTION. Europe (excluded Portugal and Ireland), Asia Minor, Transcaucasus, N-Iran, Kazakhstan, Russian Far East, and Siberia. (Italy: N, C, S, Si).

CHOROTYPE. Sibero-European.

Grapholita funebrana Treitschke, 1835

MATERIAL EXAMINED. 1 male, Monte Sant'Angelo (Foggia), Vallone Pulsano, $400 \mathrm{~m}$, 16.V.1997, leg. Mancini, Sciarretta, Trematerra.

DistRIBUTION. Two forms known. The western form occurs in Europe (except for Shetland islands and N-Scandinavia), North Africa, Asia Minor, Central Asia and S-Kazakhstan. The eastern form is recorded from Transbaikalia to China and Japan (Honsyu, Hokkaido). Artificially introduced to many territories. (Italy: N, C, S, $\mathrm{Si}, \mathrm{Sa}$ ).

CHOROTYPE. Palaearctic.

Grapholita janthinana (Duponchel, 1835)

MATERIAL EXAMINED. 5 males and 1 female, Vico del Gargano (Foggia), 558 m, 29.VII.2020, leg. Goglia and Palmieri. 1 male and 1 female, San Giovanni Rotondo (Foggia), $595 \mathrm{~m}, 25$. VIII.2020, leg. Goglia and Palmieri.

DisTRIBUTION. Europe, Asia Minor, Crimea, Transcaucasia, and Kazakhstan. (Italy: N, C, S, Si, Sa).

CHOROTYPE. Turano-European.

Pammene fasciana (Linnaeus, 1761)

MATERIAL EXAMINED. 1 male, Vico del Garganico (Foggia), 536 m, 28.VII.2020, leg. Goglia and Palmieri; 2 females, idem, $558 \mathrm{~m}, 29$.VII.2020.

DisTRIBUTION. Europe, Asia Minor, Caucasus, Transcaucasia, and Iran. (Italy: N, C, S, Si, Sa).

CHOROTYPE. Turano-European.

Pammene giganteana (Peyerimhoff, 1863)

MATERIAL EXAMINED. 3 males, Dune di Lesina (Foggia), 5 m, 28.III.1997, leg. Sciarretta.

DISTRIBUTION. Distributed in Europe from British islands, S-Scandinavia and Italy to E-Europe. (Italy: N, $\mathrm{C}, \mathrm{S}, \mathrm{Si})$.

CHOROTYPE. European.

Pammene rhediella (Clerck, 1759)

MATERIAL EXAMINED. 1 male, Monte Sant'Angelo (Foggia), 500 m, 16.V.1997, leg. Mancini, Sciarretta, Trematerra. 
DisTRIBUTION. W Palaearctic: Europe north to Scotland, S-Scandinavia and Karelia; Asia Minor, Transcaucasia, mountains of Turkmenia, and Central Asia. (Italy: N, C, S, Si).

Chorotype. Centralasiatic-European.

Dichrorampha flavidorsana Knaggs, 1867

MATERIAL EXAMINED. 1 male, Foresta Umbra, 600 m, 2.VII.1980, leg. Baldizzone.

DiSTRIBUTION. Europe, from Spain to Russia, W and S-Siberia. (Italy: N, C, S, Si).

CHOROTYPE. Sibero-European.

\section{CONSIDERATIONS ON THE TORTRICIDAE FAUNA OF GARGANO TERRITORY}

During our entomological expeditions carried out during 2019-2020 in Gargano territory, we caught 33 species of Lepidoptera Tortricidae, to which we added 34 species collected in the previous years by the senior author or by other entomologists, up to a total of 67 taxa of the family. The two most numerous tortricids subfamilies recorded are the Olethreutinae and Tortricinae, which has 37 species and 30 species, respectively; the subfamily Chlidanotinae is not represented. Species belonging to almost all the tribes cited in the Italian fauna (TREMATERRA, 2003) have been found in Gargano territory (Tortricini with 4 species, Cochylini 10, Cnephasiini 6, Archipini 10, Olethreutini 7, Enarmoniini 4, Eucosmini 12, and Grapholitini 14), with the exception of the members of the tribes Sparganothini, Euliini, Polyorthini, and Bactrini.

Ancylis minimana (Caradja, 1916) (Tortricinae Enarmoniini), listed from Eastern and SE parts of E-Europe to Ciscaucasia, Ural Mts, and Caucasus, is reported for the first time in Italy.

From a biogeographic point of view it can be noted that in Gargano territory there are few Cosmopolitan species (8.95\%), European (11.94\%), and Holarctic (13.43\%). More than $65 \%$ of the species show a wide Palaearctic distribution: in this grouping, the species with the chorotypes Asiatic-European (13.43\%), Palaearctic (11.94\%) and Turano-European $(11.94 \%)$ are well represented. Fewer are the W-Palaearctic $(8.95 \%)$, Centralasiatic-European $(7.46 \%)$, Sibero-European $(5.97 \%)$, Centralasiatic-Europeo-Mediterranean (2.99\%), Turano-EuropeoMediterranean (1.49), and Europeo-Mediterranean $(1.49 \%)$. Around $12 \%$ of the collected taxa are tortricids with European distribution; the elements belonging to this chorotype show S-European (4.47\%), European (2.99\%), European-Anatolic (2.99\%), and S-EuropeanAnatolic (1.49\%).

Overall, we observed that a limited number of specimens belonging to entities potentially harmful to agricultural and forest plants was recorded, especially in the central part of the territory (in Gargano National Park) this suggests that this area is in good health and maintains a high degree of naturalness. However, it is worth noting the negative role of grazing overload on the biodiversity of this group of Lepidoptera, whose whole larvae often inhabit the land with grass- and shrubland.

\section{ACKNOWLEDGEMENTS}

We are grateful to dr Florena Palmieri (University of Molise, Campobasso, Italy) for her help in fieldwork, and the people of the Gargano National Park for technical assistance.

\section{REFERENCES}

AARVIK L.E., 2013 - Tortricidae - In: Karsholt O. \& Van Nieukerken E.J. Fauna Europaea version 2.6.2. Available from http://www.faunaeur.org.

BIONDI E., CASAVECCHIA S., BECCARISI L., MARCHIORI S., MEDAGLi P., ZUCCARIELlO V., 2010 - La vegetazione d'Italia. - Palombi Editori, Roma: 1-409.

BRown J.W., 2005 - World Catalogue of Insects. Volume 5. Tortricidae (Lepidoptera). - Apollo Books. Stenstrup: 1-741.

EUROPEAN COMMISSION, 1992 - Council Directive 92/43 CEE on the conservation of natural habitats and of wild fauna and flora. - Europen Community Gazzette, 206: 1-50.

FENAROLI L., 1966 - Il Gargano, suoi aspetti vegetazionali e floristici. - Annali Accademia Italiana di Scienze Forestali, 15: 107-135.

GILligan T.M., BAIXERAS J., BROWN J.W., 2018 T@RTS: Online World Catalogue of the Tortricidae (Ver. 4.0). http://www.tortricid.net/catalogue.asp

PARENZAN P., 1994 - Proposta di codificazione per una gestione informatica dei corotipi W-Paleartici, con particolare riferimento alla fauna italiana. - Entomologica, 28: 93-98.

PERRINO E.V., WAGENSOMMER R.P., 2012 - Aggiornamenti floristici per il Gargano (Puglia) con riferimento agli habitat della Direttiva 92/43/EEC. - Informatore Botanico Italiano, 44: 163-170.

RAZOWSKI J., 2002 - Tortricidae of Europe. Volume 1. Tortricinae and Chlidanotinae. - František Slamka. Bratislava: 1-247.

RAZOWSKI J., 2003 - Tortricidae of Europe. Volume 2. Olethreutinae. - František Slamka. Bratislava: 1-301.

TREMATERRA P., 2003 - Catalogo dei Lepidoptera Tortricidae della fauna italiana: geonemia, distribuzione in Italia, note biologiche, identificazione. - Bollettino di Zoologia agraria e di Bachicoltura, Ser. II, 35 (suppl. 1): 1-270.

TREMATERrA P., Sciarretta A., PARENZAN P., 1997 Note faunistiche su alcuni interessanti Tortricidi dell'Italia meridionale (Lepidoptera). - Entomologica, 31: 181-189.

Vigna Taglianti A., Audisio P.A., Belfiore C., Biondi M.A., Bologna M.A., CARPANETO G.M., DE Biase A., De Felici S., Piattella E., Racheli T., ZAPPAROLI M., ZOIA S., 1992 - Riflessioni di gruppo sui corotipi fondamentali della fauna W-paleartica ed in particolare italiana. - Biogeographia, 16: 159-210.

ZANGHERI S., 1956 - Le attuali conoscenze sui Lepidotteri del promontorio del Gargano e delle Isole Tremiti, con osservazioni sulle specie a distribuzione transadriatica. - Memorie di Biogeografia Adriatica, 3: 245-298. 